\title{
Application of Adomian's Decomposition Method for the Analytical Solution of Space Fractional Diffusion Equation
}

\author{
Mehdi Safari ${ }^{1}$, Mohammad Danesh ${ }^{2}$ \\ ${ }^{1}$ Department of Mechanical Engineering, Islamic Azad University, Aligoodarz Branch, Aligoodarz, Iran \\ ${ }^{2}$ Department of Mechanical Engineering, Isfahan University of Technology, Isfahan, Iran \\ E-mail:ms_safari2005@yahoo.com \\ Received July 1, 2011; revised September 26, 2011; accepted October 5, 2011
}

\begin{abstract}
Spatially fractional order diffusion equations are generalizations of classical diffusion equations which are increasingly used in modeling practical super diffusive problems in fluid flow, finance and others areas of application. This paper presents the analytical solutions of the space fractional diffusion equations by Adomian's decomposition method (ADM). By using initial conditions, the explicit solutions of the equations have been presented in the closed form. Two examples, the first one is one-dimensional and the second one is two-dimensional fractional diffusion equation, are presented to show the application of the present techniques. The present method performs extremely well in terms of efficiency and simplicity.
\end{abstract}

Keywords: Adomian’s Decomposition Method, Fractional Derivative, Fractional Diffusion Equation

\section{Introduction}

Fractional diffusion equations are used to model problems in Physics [1-3], Finance [4-7], and Hydrology [812]. Fractional space derivatives may be used to formulate anomalous dispersion models, where a particle plume spreads at a rate that is different than the classical Brownian motion model. When a fractional derivative of order $1<\alpha<2$ replaces the second derivative in a diffusion or dispersion model, it leads to a super diffusive flow model. Nowadays, fractional diffusion equation plays important roles in modeling anomalous diffusion and subdiffusion systems, description of fractional random walk, unification of diffusion and wave propagation phenomenon, see, e.g. the reviews in [1-16], and references therein. Consider a one-dimensional fractional diffusion equation considered in [17]

$$
\frac{\partial u(x, t)}{\partial t}=d(x) \frac{\partial^{\alpha} u(x, t)}{\partial x^{\alpha}}+q(x, t),
$$

on a finite domain $x_{L}<x<x_{R}$ with $1<\alpha \leq 2$. We assume that the diffusion coefficient (or diffusivity) $d(x)$ $>0$. We also assume an initial condition $u(x, t=0)=s(x)$ for $x_{L}<x<x_{R}$ and Dirichlet boundary conditions of the form $u\left(x_{L}, t\right)=0$ and $u\left(x_{R}, t\right)=b_{R}(t)$. Equation
(1) uses a Riemann fractional derivative of order $\alpha$.

Consider a two-dimensional fractional diffusion equation considered in [18]

$$
\begin{aligned}
\frac{\partial u(x, y, t)}{\partial t}= & d(x, y) \frac{\partial^{\alpha} u(x, y, t)}{\partial x^{\alpha}}+e(x, y) \frac{\partial^{\beta} u(x, y, t)}{\partial x^{\beta}}, \\
& +q(x, y, t)
\end{aligned}
$$

on a finite rectangular domain $x_{L}<x<x_{H}$ and $y_{L}<y<y_{R}$, with fractional orders $1<\alpha \leq 2$ and $1<\beta \leq 2$, where the diffusion coefficients $d(x, y)>0$ and $e(x, y)>0$. The 'forcing' function $q(x, y, t)$ can be used to represent sources and sinks. We will assume that this fractional diffusion equation has a unique and sufficiently smooth solution under the following initial and boundary conditions. Assume the initial condition $u(x, y$, $t=0)=f(x, y)$ for $x_{L}<x<x_{H}$ and $y_{L}<y<y_{R}$, and Dirichlet boundary condition $u(x, y, t)=B(x, y, t)$ on the boundary (perimeter) of the rectangular region

$x_{L}<x<x_{H}, y_{L}<y<y_{R}$, with the additional restriction that $B\left(x_{L}, y, t\right)=B\left(x, y_{L}, t\right)=0$. In physical applications, this means that the left/lower boundary is set far away enough from an evolving plume that no significant concentrations reach that boundary. The classical dispersion equation in two-dimensions is given by $\alpha=\beta=2$. 
The values of $1<\alpha \leq 2$ and $1<\beta \leq 2$ model a super diffusive process in that coordinate. Equation (2) also uses Riemann fractional derivatives of order $\alpha$ and $\beta$. In this paper, we use the Adomian's decomposition method (ADM) to obtain the solutions of the fractional diffusion Equations (1) and (2). Large classes of linear and nonlinear differential equations, both ordinary as well as partial, can be solved by the ADM. The decomposition method provides an effective procedure for analytical solution of a wide and general class of dynamical systems representing real physical problems [19-25]. This method efficiently works for initial-value or boundary-value problems and for linear or nonlinear, ordinary or partial differential equations and even for stochastic systems. Moreover, we have the advantage of a single global method for solving ordinary or partial differential equations as well as many types of other equations. Recently, the solution of fractional differential equation has been obtained through ADM by the researchers [26-28]. The application of ADM for the solution of nonlinear fractional differential equations has also been established by Shawagfeh, Saha Ray and Bera [27, 28]. However, we use the ADM to solve fractional diffusion Equations (1) and (2) and finally the results are illustrated in graphical figures.

\section{Mathematical Aspects}

The mathematical definition of fractional calculus has been the subject of several different approaches [29,30]. The most frequently encountered definition of an integral of fractional order is the Riemann-Liouville integral, in which the fractional order integral is defined as

$$
D_{t}^{-q} f(t)=\frac{\mathrm{d}^{-q} f(t)}{\mathrm{d} t^{-q}}=\frac{1}{\Gamma(q)} \int_{0}^{t} \frac{f(t) \mathrm{d} x}{(t-x)^{1-q}},
$$

while the definition of fractional order derivative is

$$
\begin{aligned}
D_{t}^{q} f(t) & =\frac{\mathrm{d}^{n}}{\mathrm{~d} t^{n}}\left(\frac{\mathrm{d}^{-(n-q)} f(t)}{\mathrm{d} t^{-(n-q)}}\right) \\
& =\frac{1}{\Gamma(n-q)} \frac{\mathrm{d}^{n}}{\mathrm{~d} t^{n}} \int_{0}^{t} \frac{f(t) \mathrm{d} x}{(t-x)^{1-n+q}},
\end{aligned}
$$

where $q(q>0$ and $q \in R)$ is the order of the operation and $\mathrm{n}$ is an integer that satisfies $n-1 \leq q<n$.

\section{Basic Idea of Adomian's Decomposition Method}

We begin with the equation

$$
L u+R(u)+F(u)=g(t),
$$

where $L$ is the operator of the highest-ordered derivatives with respect to $t$ and $R$ is the remainder of the linear operator. The nonlinear term is represented by $F(u)$. Thus we get

$$
L u=g(t)-R(u)-F(u),
$$

The inverse $L^{-1}$ is assumed an integral operator given by

$$
L_{t}^{-1}=\int_{0}^{t}(\cdot) \mathrm{d} t,
$$

The operating with the operator $L^{-1}$ on both sides of Equation (6) we have

$$
u=f_{0}+L^{-1}(g(t)-R(u)-F(u)),
$$

where $f_{0}$ is the solution of homogeneous equation

$$
L u=0 \text {, }
$$

involving the constants of integration. The integration constants involved in the solution of homogeneous Equation (9) are to be determined by the initial or boundary condition according as the problem is initialvalue problem or boundary-value problem.

The ADM assumes that the unknown function $u(x, t)$ can be expressed by an infinite series of the form

$$
u(x, t)=\sum_{n=0}^{\infty} u_{n}(x, t),
$$

and the nonlinear operator $F(u)$ can be decomposed by an infinite series of polynomials given by

$$
F(u)=\sum_{n=0}^{\infty} A_{n},
$$

where $u_{n}(x, t)$ will be determined recurrently, and $A_{n}$ are the so-called polynomials of $u_{0}, u_{1}, \cdots, u_{n}$ defined by

$$
A n=\frac{1}{n !} \frac{\mathrm{d}^{n}}{\mathrm{~d} \lambda^{n}}\left[F\left(\sum_{n=0}^{\infty} \lambda^{i} u_{i}\right)\right]_{\lambda=0}, n=0,1,2, \cdots,
$$

\section{The Fractional Diffusion Equation Model and Its Solution by ADM}

We adopt Adomian decomposition method for solving Equation (1). In the light of this method we assume that

$$
u=\sum_{n=0}^{\infty} u_{n},
$$

to be the solution of Equation (1). Now, Equation (1) can be rewritten as

$$
L_{t} u(x, t)=d(x) D_{x}^{\alpha}(x, t)+q(x, t),
$$

where $L_{t}=\frac{\partial}{\partial t}$ which is an easily invertible linear operator, $D_{x}^{\alpha}(\cdot)$ is the Riemann-Liouville derivative of order 
$\alpha$.Therefore, by Adomian decomposition method, we can write,

$$
u(x, t)=u(x, 0)+L_{t}^{-1}\left(d(x) D_{x}^{\infty}\left(\sum_{n=0}^{\infty} u_{n}\right)\right)+L_{t}^{-1}(q(x, t)),
$$

Each term of series (13) is given by Adomian decomposition method recurrence relation

$$
\begin{gathered}
u_{0}=f, \\
u_{n+1}=L_{t}^{-1}\left(d(x) D_{x}^{\alpha} u_{n}\right), n \geq 0,
\end{gathered}
$$

where $f=u(x, 0)+L_{t}^{-1}(q(x, t))$.

It is worth noting that once the zeroth component $u_{0}$ is defined, then the remaining components $u_{n}, n \geq 1$ can be completely determined; each term is computed by using the previous term. As a result, the components $u_{0}, u_{1}, \cdots$ are identified and the series solutions thus entirely determined. However, in many cases the exact solution in a closed form may be obtained.

Similarly, for Equation (2) using Adomian decomposition method, we can obtain

$$
\begin{aligned}
u(x, y, t)= & u(x, y, 0)+L_{t}^{-1}\left(d(x, y) D_{x}^{\alpha}\left(\sum_{n=0}^{\infty} u_{n}\right)\right) \\
& +L_{t}^{-1}\left(e(x, y) D_{y}^{\beta}\left(\sum_{n=0}^{\infty} u_{n}\right)\right)+L_{t}^{-1}(q(x, y, t))
\end{aligned}
$$

The Adomian decomposition method recurrence scheme is

$$
\begin{aligned}
& u_{0}=f, \\
& u_{n+1}=L_{t}^{-1}\left(d(x, y) D_{x}^{\alpha} u_{n}\right)+L_{t}^{-1}\left(e(x, y) D_{y}^{\beta} u_{n}\right), \\
& n \geq 0,
\end{aligned}
$$

where $f=u(x, y, 0)+L_{t}^{-1}(q(x, y, t))$.

\section{Numerical Illustration}

\subsection{Example 1}

Let us consider a one-dimensional fractional diffusion equation for the Equation (1), as taken in [17]

$$
\frac{\partial u(x, t)}{\partial t}=d(x) \frac{\partial^{1.8} u(x, t)}{\partial x^{1.8}}+q(x, t),
$$

on a finite domain $0<x<1$, with the diffusion coefficient

$$
d(x)=\Gamma(2.2) x^{2.8} / 6=0.183634 x^{2.8},
$$

the source/sink function

$$
q(x, t)=-(1+x) \mathrm{e}^{-t} x^{3},
$$

the initial condition

$$
u(x, 0)=x^{3}, \text { for } 0<x<1,
$$

and the boundary conditions

$$
u(0, t)=0, u(1, t)=\mathrm{e}^{-t}, \text { for } t>0,
$$

Implementation of Adomian's Decomposition Method Equation (21) can be rewritten in operator form as

$$
L_{t} u(x, t)=d(x) D_{x}^{1.8} u(x, t)+q(x, t),
$$

where $L_{t}=\frac{\partial}{\partial t}$ symbolizes the easily invertible linear differential operator, $D_{x}^{1.8}(\bullet)$ is the Riemann-Liouville derivative of order 1.8 . If the invertible operator

$L_{t}^{-1}=\int_{0}^{t}(\cdot) \mathrm{d} t$ is applied to Equation (26), then

$$
L_{t}^{-1} L_{t} u(x, t)=L_{t}^{-1}\left(d(x) D_{x}^{1.8} u(x, t)+q(x, t)\right),
$$

is obtained. By this

$$
u(x, t)=u(x, 0)+L_{t}^{-1}\left(d(x) D_{x}^{1.8} u(x, t)+q(x, t)\right),
$$

is found. Here the main point is that the solution of the decomposition method is in the form of

$$
u(x, t)=\sum_{n=0}^{\infty} u_{n}(x, t),
$$

Substituting from Equation (29) into Equation (28), we find

$$
\begin{aligned}
& \sum_{n=0}^{\infty} u_{n}(x, t)=u(x, 0) \\
& \quad+L_{t}^{-1}\left(d(x) D_{x}^{1.8}\left(\sum_{n=0}^{\infty} u_{n}(x, t)\right)+q(x, t)\right)
\end{aligned}
$$

is found. Thus according to Equation (7) approximate solution can be obtained as follows:

$$
\begin{gathered}
u_{0}(x, t)=x^{3}, \\
u_{1}(x, t)=-x^{3}-x^{4}+1.000001369 x^{4} t+\mathrm{e}^{-t}\left(x^{3}+x^{4}\right), \\
u_{2}(x, t)=\int_{0}^{t}\left(d(x) D_{x}^{1.8} u_{1}(x, t)+q(x, t)\right) \mathrm{d} t,
\end{gathered}
$$

The approximate solution of the one-dimensional fractional diffusion equation is obtained as

$$
u(x, t)=u_{0}(x, t)+u_{1}(x, t)+u_{2}(x, t),
$$

In Figure 1 we can see the 3-D result of approximate solution of the one-dimensional fractional diffusion equation by ADM. 


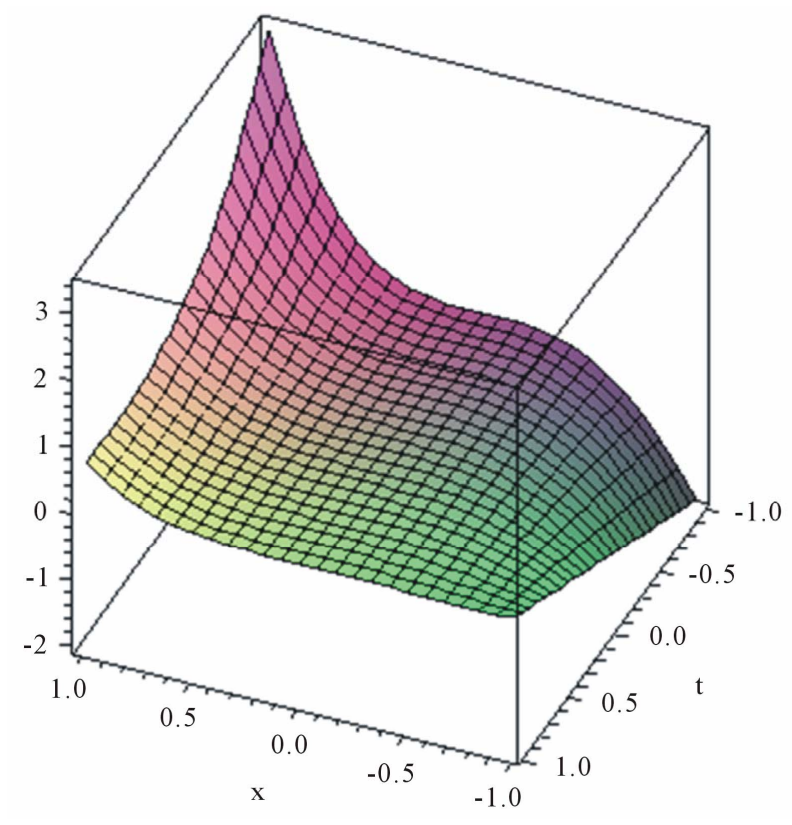

Figure 1. For the one-dimensional fractional diffusion equation with the initial condition (24) of Equation (21), ADM result for $u(x, t)$.

\subsection{Example 2}

Let us consider a two-dimensional fractional diffusion equation for the Equation (2), considered in [18].

$$
\begin{aligned}
\frac{\partial u(x, y, t)}{\partial t}= & d(x, y) \frac{\partial^{1.8} u(x, y, t)}{\partial x^{1.8}} \\
& +e(x, y) \frac{\partial^{1.6} u(x, y, t)}{\partial y^{1.6}}+q(x, y, t),
\end{aligned}
$$

on a finite rectangular domain $0<x<1,0<y<1$, for 0 $<t<T_{\text {end }}$ with the diffusion coefficients

$$
d(x, y)=\Gamma(2.2) x^{2.8} y / 6,
$$

and

$$
e(x, y)=2 x^{2.6} y / \Gamma(4.6),
$$

and the forcing function

$$
q(x, y, t)=-(1+2 x y) \mathrm{e}^{-t} x^{3} y^{3.6},
$$

with the initial condition

$$
u(x, y, 0)=x^{3} y^{3.6},
$$

and Dirichlet boundary conditions on the rectangle in the form $u(x, 0, t)=u(0, y, t)=0, u(x, 1, t)=\mathrm{e}^{-t} x^{3}$, and $u(1, y, t)=\mathrm{e}^{t} y^{3.6}$, for all $t \geq 0$.

\section{Implementation of Adomian's Decomposition Method} Now, Equation (35) can be rewritten in operator form as

$$
\begin{aligned}
L_{t} u(x, y, t)= & d(x, y) D_{x}^{1.8}(x, y, t) \\
& +e(x, y, t) D_{y}^{1.6} u(x, y, t)+q(x, y, t),
\end{aligned}
$$

where $L_{t}=\frac{\partial}{\partial t}$ symbolizes the easily invertible linear differential operator, $D_{x}^{1.8}(\bullet)$ and $D_{y}^{1.6}(\bullet)$ and are the Riemann-Liouville derivatives of order 1.8 and 1.6, respectively.

If the invertible operator $L_{t}^{-1}=\int_{0}^{t}(\cdot) \mathrm{d} t$ is applied to Equation (40), then

$$
\begin{aligned}
L_{t}^{-1} L_{t} u(x, y, t)= & L_{t}^{-1}\left(d(x, y) D_{x}^{1.8} u(x, y, t)\right. \\
& \left.+e(x, y, t) D_{y}^{1.6} u(x, y, t)+q(x, y, t)\right),
\end{aligned}
$$

is obtained. By this

$$
\begin{aligned}
u(x, y, t)= & u(x, y, 0)+L_{t}^{-1}\left(d(x, y) D_{x}^{1.8} u(x, y, t)\right. \\
& \left.+e(x, y, t) D_{y}^{1.6} u(x, y, t)+q(x, y, t)\right),
\end{aligned}
$$

is found. Here the main point is that the solution of the decomposition method is in the form of

$$
u(x, y, t)=\sum_{n=0}^{\infty} u_{n}(x, y, t),
$$

Substituting from Equation (43) into Equation (42), we find

$$
\begin{aligned}
& \sum_{n=0}^{\infty} u_{n}(x, y, t)=u(x, y, 0) \\
& \quad+L_{t}^{-1}\left(d(x, y) D_{x}^{1.8} \sum_{n=0}^{\infty} u_{n}(x, y, t)\right. \\
& \left.\quad+e(x, y, t) D_{y}^{1.6} \sum_{n=0}^{\infty} u_{n}(x, y, t)+q(x, y, t)\right),
\end{aligned}
$$

is found.

Thus according to Equation (7) approximate solution can be obtained as follows:

$$
u_{0}(x, y, t)=x^{3} y^{3.6},
$$

$$
\begin{aligned}
u_{1}(x, y, t)= & -x^{3} y^{\frac{18}{5}}-2 x^{4} y^{\frac{23}{5}}+2 x^{4} y^{\frac{23}{5}} t \\
& +\mathrm{e}^{-t} x^{3} y^{\frac{18}{5}}+2 \mathrm{e}^{-t} x^{4} y^{\frac{23}{5}}
\end{aligned}
$$

$$
\begin{aligned}
u_{2}(x, y, t)= & \int_{0}^{t}\left(d(x, y) D_{x}^{1.8} u_{1}(x, y, t)\right. \\
& \left.+e(x, y, t) D_{y}^{1.6} u_{1}(x, y, t)+q(x, y, t)\right) \mathrm{d} t,
\end{aligned}
$$

The approximate solution of the two-dimensional fractional diffusion equation is obtained as

$$
u(x, t)=u_{0}(x, t)+u_{1}(x, t)+u_{2}(x, t),
$$

In Figure 2 we can see the 3-D result of approximate 


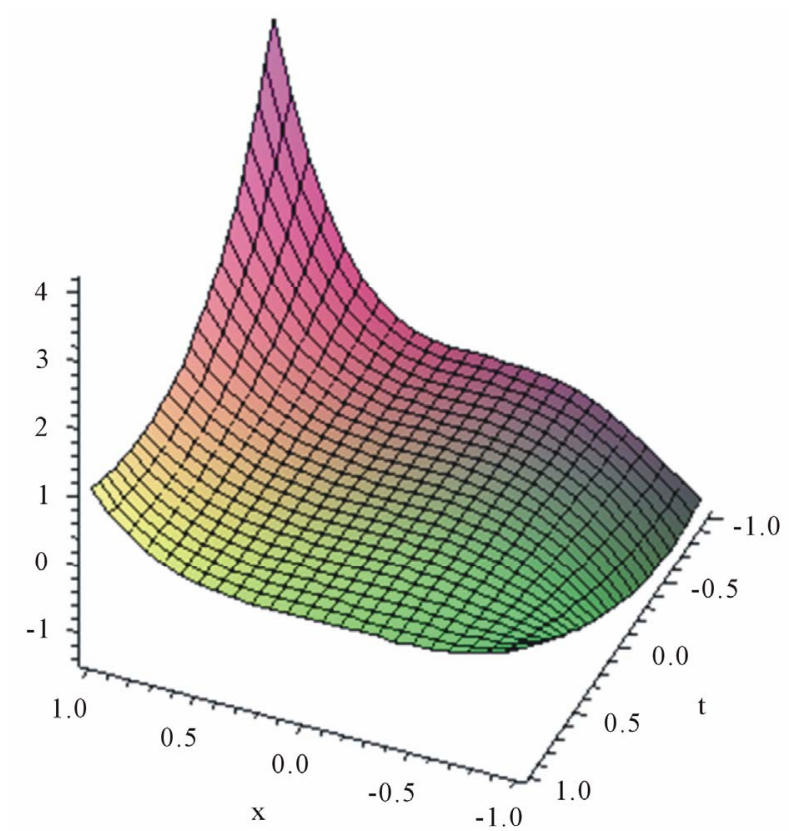

Figure 2. For the two-dimensional fractional diffusion equation with the initial condition (39) of Equation (35), ADM result for $u(x, t)$ with $y=1$.

solution of the two-dimensional fractional diffusion equation by ADM.

\section{Conclusions}

In this paper, Adomian's decomposition method has been successfully applied to find the solution of space fractional diffusion equation. All cases show that the results of the ADM method are very good and the obtained solutions are shown graphically. In our work, we use the Maple Package to calculate the functions obtained from the Adomian's decomposition method.

\section{References}

[1] R. Metzler, E. Barkai and J. Klafter, “Anomalous Diffusion and Relaxation Close to Thermal Equilibrium: A Fractional Fokker-Planck Equation Approach,” Physics Review Letters, Vol. 82, No. 18, 1999, pp. 3563-3567. doi:10.1103/PhysRevLett.82.3563

[2] R. Metzler and J. Klafter, “The Random Walk's Guide to Anomalous Diffusion: A Fractional Dynamics Approach,” Physics Reports, Vol. 339, No. 1, 2000, pp. 1-77. doi:10.1016/S0370-1573(00)00070-3

[3] R. Metzler and J. Klafter, "The Restaurant at the End of the Random Walk: Recent Developments in the Description of Anomalous Transport by Fractional Dynamics," Journal of Physics A, Vol. 37, No. 31, 2004, pp. 161-208. doi:10.1088/0305-4470/37/31/R01

[4] R. Gorenflo, F. Mainardi, E. Scalas and M. Raberto,
"Fractional Calculus and Continuous-Time Finance. III. The Diffusion Limit,” Mathematical Finance. Konstanz, 2000, Trends Math Birkhuser Basel, 2001, pp. 171-180.

[5] F. Mainardi, M. Raberto, R. Gorenflo and E. Scalas, "Fractional Calculus and Continuous-Time Finance II: The Waiting-Time Distribution,” Physica A, Vol. 287, No. 3-4, 2000, pp. 468-481. doi:10.1016/S0378-4371(00)00386-1

[6] E. Scalas, R. Gorenflo and F. Mainardi, "Fractional Calculus and Continuous-Time Finance," Physica A, Vol. 284, No. 1-4, 2000, pp. 376-384. doi:10.1016/S0378-4371(00)00255-7

[7] M. Raberto, E. Scalas and F. Mainardi, "Waiting-Times and Returns in High Frequency Financial Data: An Empirical Study,” Physica A, Vol. 314, No. 1-4, 2002, pp. 749-755. doi:10.1016/S0378-4371(02)01048-8

[8] D. A. Benson, S. Wheatcraft and M. M. Meerschaert, "Application of a Fractional Advection Dispersion Equation,” Water Resource Research, Vol. 36, No. 6, 2000, pp. 1403-1412. doi:10.1029/2000WR900031

[9] B. Baeumer, M. M. Meerschaert, D. A. Benson and S. W. Wheatcraft, "Subordinated Advection-Dispersion Equation for Contaminant Transport," Water Resource Research, Vol. 37, No. 6, 2001, pp. 1543-1550. doi:10.1029/2000WR900409

[10] D. A. Benson, R. Schumer, M. M. Meerschaert and S. W. Wheatcraft, "Fractional Dispersion, Lévy Motions, and the MADE Tracer Tests," Transport in Porous Media, Vol. 42, No. 1-2, 2001, pp. 211-240. doi:10.1023/A:1006733002131

[11] R. Schumer, D. A. Benson, M. M. Meerschaert and S. W. Wheatcraft, "Eulerian Derivation of the Fractional Advection-Dispersion Equation,” Journal of Contaminant Hydrology, Vol. 48, No. 1-2, 2001, pp. 69-88. doi:10.1016/S0169-7722(00)00170-4

[12] R. Schumer, D. A. Benson, M. M. Meerschaert and B. Baeumer, "Multiscaling Fractional Advection-Dispersion Equations and Their Solutions," Water Resource Research, Vol. 39, No. 1, 2003, pp. 1022-1032. doi:10.1029/2001WR001229

[13] A. Carpinteri and F. Mainardi (Eds.), "Fractals and Fractional Calculus in Continuum Mechanics," SpringerVerlag, New York, 1997, pp. 291-348.

[14] F. Mainardi and G. Pagnini, "The Wright Functions as Solutions of the Time Fractional Diffusion Equations," Applied Mathematics and Computation, Vol. 141, No. 1, 2003, pp. 51-62. doi:10.1016/S0096-3003(02)00320-X

[15] O. P. Agrawal, "Solution for a Fractional Diffusion-Wave Equation Defined in a Bounded Domain,” Nonlinear Dynamics, Vol. 29, No. 1-4, 2002, pp. 145-155. doi:10.1023/A:1016539022492

[16] W. R. Schneider and W. Wyss, "Fractional Diffusion and Wave Equations," Journal of Mathematical Physics, Vol. 30, No. 1, 1989, pp. 134-144. doi:10.1063/1.528578

[17] M. M. Meerschaert, H. Scheffler and C. Tadjeran, "Finite Difference Methods for Two-Dimensional Fractional Dispersion Equation,” Journal of Computational Physics, 
Vol. 211, No. 1, 2006, pp. 249-261. doi:10.1016/j.jcp.2005.05.017

[18] C. Tadjeran, M. M. Meerschaert and H. Scheffler, "Finite Difference Methods for Two-Dimensional Fractional Dispersion Equation,” Journal of Computational Physics, Vol. 213, No. 1, 2006, pp. 205-213. doi:10.1016/j.jcp.2005.08.008

[19] G. Adomian, "An Analytical Solution of the Stochastic Navier-Stokes System," Foundations of Physics, Vol. 21, No. 7, 1991, pp. 831-843. doi:10.1007/BF00733348

[20] G. Adomian and R. Rach, "Linear and Nonlinear Schrödinger Equations,” Foundations of Physics, Vol. 21, No. 8, 1991, pp. 983-991. doi:10.1007/BF00733220

[21] G. Adomian, "Solution of Physical Problems by Decomposition," Computers and Mathematics with Applications, Vol. 27, No. 9-10, 1994, pp. 145-154. doi:10.1016/0898-1221(94)90132-5

[22] G. Adomian, "Solutions of Nonlinear PDE," Applied Mathematics Letters, Vol. 11, No. 3, 1998, pp. 121-123. doi:10.1016/S0893-9659(98)00043-3

[23] D. D. Ganji, M. Safari and R. Ghayor, "Application of He's Variational Iteration Method and Adomian's Decomposition Method to Sawada-Kotera-Ito SeventhOrder Equation,” Numerical Methods for Partial Differential Equations, Vol. 27, No. 4, 2011, pp. 887-897. doi:10.1002/num.20559

[24] M. Safari, D. D. Ganji and M. Moslemi, “Application of He's Variational Iteration Method and Adomian's Decomposition Method to the Fractional KdV-BurgersKuramoto Equation,” Computers and Mathematics with
Applications, Vol. 58, No. 11-12, 2009, pp. 2091-2097. doi:10.1016/j.camwa.2009.03.043

[25] D. D. Ganji, E. M. M. Sadeghi and M. Safari, “Application of He's Variational Iteration Method and Adomian's Decomposition Method to Prochhammer Chree Equation," International Journal of Modern Physics $B$, Vol. 23, No. 3, 2009, pp. 435-446. doi:10.1142/S0217979209049656

[26] A. J. George and A. Chakrabarti, "The Adomian Method Applied to Some Extraordinary Differential Equations," Applied Mathematics Letters, Vol. 8, No. 3, 1995, pp. 91-104. doi:10.1016/0893-9659(95)00036-P

[27] N. T. Shawagfeh, "Analytical Approximate Solutions for Nonlinear Fractional Differential Equations,” Applied Mathematics and Computation, Vol. 131, No. 2, 2002, pp. 517-529. doi:10.1016/S0096-3003(01)00167-9

[28] S. Saha Ray and R. K. Bera, “Analytical Solution of a Dynamic System Containing Fractional Derivative of Order One-Half by Adomian Decomposition Method," Applied Mathematics and Computation, Vol. 167, No. 1, 2005, pp. 561-571.

[29] I. Podlubny, “An Introduction to Fractional Derivatives, Fractional Differential Equations, Some Methods of Their Solution and Some of Their Applications," Fractional Differential Equations, Mathematics in Science and Engineering, Vol. 198, Academic Press, San Diego, 1999.

[30] K. B. Oldham and J. Spanier, "Fractional Calculus: Theory and Applications, Differentiation and Integration to Arbitrary Order," Academic Press, Inc., New YorkLondon, 1974, 234 Pages. 